\title{
LIGHTLIKE SUBMANIFOLDS OF A SEMI-RIEMANNIAN MANIFOLD WITH A SEMI-SYMMETRIC NON-METRIC CONNECTION
}

\author{
DAE Ho JiN
}

\begin{abstract}
We study lightlike submanifolds $M$ of a semi-Riemannian manifold $\bar{M}$ with a semi-symmetric non-metric connection subject to the conditions; (a) the characteristic vector field of $\bar{M}$ is tangent to $M$, (b) the screen distribution on $M$ is totally umbilical in $M$ and (c) the co-screen distribution on $M$ is conformal Killing.
\end{abstract}

\section{INTRODUCTION}

In the classical theory of spacetime, while the rest spaces of timelike curves are spacelike subspaces of the tangent spaces, the rest spaces of null curves are lightlike subspaces of the tangent spaces [9]. To investigate this, Hawking and Ellis introduced the notion of so-called screen spaces in section 4.2 of their book [5]. As for any semiRiemannian manifold there is a natural existence of lightlike subspaces, in a 1996 book [3] Duggal-Bejancu published their work on the general theory of degenerate (lightlike) submanifolds to fill a gap in the study of submanifolds. Since then there has been very active study on lightlike geometry of submanifolds. The geometry of lightlike submanifolds is used in mathematical physics, in particular, in general relativity since lightlike submanifolds produce models of different types of horizons (event horizons, Cauchy's horizons, Kruskal's horizons). Lightlike hypersurfaces are also studied in the theory of electromagnetism [3].

Ageshe and Chafle [1] introduced the notion of a semi-symmetric non-metric connection on a Riemannian manifold. Although now we have lightlike version of a large variety of Riemannian submanifolds, a general notion of lightlike submanifolds of a semi-Riemannian manifold with a semi-symmetric non-metric connection has not

Received by the editors January 26, 2012. Revised August 6, 2012. Accepted Aug. 13, 2012. 2000 Mathematics Subject Classification. 53C25, 53C40, 53C50.

Key words and phrases. totally umbilical, conformal Killing distribution, irrotational, semiRiemannian manifold with a semi-symmetric non-metric connection. 
been introduced as yet. Only there are some limited papers on particular subcases recently studied by Yaşar, Çöken and Yücesan [10], Jin [6] and Jin and Lee [7].

Motivated by the notion of a semi-symmetric non-metric connection on a Riemannian manifold, the objective of this article is to study the geometry of lightlike submanifolds $M$ of a semi-Riemannian manifold $\bar{M}$ with a semi-symmetric nonmetric connection. In section 2 , we recall some of fundamental formulas in the theory of such a lightlike submanifold and prove some results which will be used in the rest of this article. In section 3, we find the condition that $M$ has an induced symmetric Ricci tensor. In section 4, we study irrotational lightlike submanifolds of a semi-Riemannian space form $\bar{M}(c)$ with a semi-symmetric non-metric connection subject to the conditions; (a) the characteristic vector field of $\bar{M}$ is tangent to $M$, (b) the screen distribution on $M$ is totally umbilical in $M$ and (c) the co-screen distribution on $M$ is conformal Killing.

\section{Semi-Symmetric Non-metric Connections}

Let $(\bar{M}, \bar{g})$ be an $(m+n)$-dimensional semi-Riemannian manifold. A connection $\bar{\nabla}$ on $\bar{M}$ is called a semi-symmetric non-metric connection [1] if, for any vector fields $X, Y$ and $Z$ on $\bar{M}, \bar{\nabla}$ and its torsion tensor $\bar{T}$ satisfy

$$
\begin{gathered}
\left(\bar{\nabla}_{X} \bar{g}\right)(Y, Z)=-\pi(Y) \bar{g}(X, Z)-\pi(Z) \bar{g}(X, Y), \\
\bar{T}(X, Y)=\pi(Y) X-\pi(X) Y,
\end{gathered}
$$

where $\pi$ is a 1 -form associated with a non-zero vector field $\zeta$ by $\pi(X)=\bar{g}(X, \zeta)$. We say that $\zeta$ is the characteristic vector field of $\bar{M}$. In the entire discussion of this article, we shall assume $\zeta$ to be unit spacelike vector field.

Let $(M, g)$ be an $m$-dimensional lightlike submanifold of $\bar{M}$. The radical distribution $\operatorname{Rad}(T M)=T M \cap T M^{\perp}$ is a vector subbundle of the tangent bundle $T M$ and the normal bundle $T M^{\perp}$, of rank $r(1 \leq r \leq \min \{m, n\})$. Then, in general, there exist two complementary non-degenerate distributions $S(T M)$ and $S\left(T M^{\perp}\right)$ of $\operatorname{Rad}(T M)$ in $T M$ and $T M^{\perp}$ respectively, which called the screen distribution and co-screen distribution on $M$, such that

$$
T M=\operatorname{Rad}(T M) \oplus_{\text {orth }} S(T M) ; T M^{\perp}=\operatorname{Rad}(T M) \oplus_{\text {orth }} S\left(T M^{\perp}\right),
$$

where $\oplus_{\text {orth }}$ denotes the orthogonal direct sum. We denote such a lightlike submanifold by $\left(M, g, S(T M), S\left(T M^{\perp}\right)\right)$. Denote by $F(M)$ the algebra of smooth functions on $M$ and by $\Gamma(E)$ the $F(M)$ module of smooth sections of a vector bundle $E$ over 
$M$. Let $\operatorname{tr}(T M)$ and $\operatorname{lt}(T M)$ be complementary (but not orthogonal) vector bundles to $T M$ in $T \bar{M}_{\mid M}$ and $T M^{\perp}$ in $S(T M)^{\perp}$ respectively and let $\left\{N_{1}, \ldots, N_{r}\right\}$ be a lightlike basis of $\operatorname{lt}(T M)[3]$ such that

$$
\bar{g}\left(N_{i}, \xi_{j}\right)=\delta_{i j}, \quad \bar{g}\left(N_{i}, N_{j}\right)=\bar{g}\left(X, N_{i}\right)=\bar{g}\left(W, N_{i}\right)=0,
$$

for all $X \in \Gamma(S(T M))$ and $W \in \Gamma\left(S\left(T M^{\perp}\right)\right)$, where the set $\left\{\xi_{1}, \cdots, \xi_{r}\right\}$ is a lightlike basis of $\operatorname{Rad}(T M)$. Then the tangent bundle $T \bar{M}$ is decomposed as follow:

$$
\begin{aligned}
T \bar{M} & =T M \oplus \operatorname{tr}(T M)=\{\operatorname{Rad}(T M) \oplus \operatorname{tr}(T M)\} \oplus_{\text {orth }} S(T M) \\
& =\{\operatorname{Rad}(T M) \oplus \operatorname{ltr}(T M)\} \oplus_{\text {orth }} S(T M) \oplus_{\text {orth }} S\left(T M^{\perp}\right) .
\end{aligned}
$$

We say that a lightlike submanifold $M \equiv\left(M, g, S(T M), S\left(T M^{\perp}\right)\right)$ of $\bar{M}$ is

(1) $r$-lightlike if $1 \leq r<\min \{m, n\}$;

(2) co-isotropic if $1 \leq r=n<m$;

(3) isotropic if $1 \leq r=m<n$;

(4) totally lightlike if $1 \leq r=m=n$.

The above three classes $(2) \sim(4)$ are particular cases of the class (1) as follows: $S\left(T M^{\perp}\right)=\{0\}, S(T M)=\{0\}$ and $S(T M)=S\left(T M^{\perp}\right)=\{0\}$ respectively. The geometry of $r$-lightlike submanifolds is more general form than that of the other three type submanifolds. For this reason, we consider only $r$-lightlike submanifolds $M$, with the following local quasi-orthonormal field of frames of $\bar{M}$ :

$$
\left\{\xi_{1}, \cdots, \xi_{r}, N_{1}, \cdots, N_{r}, F_{r+1}, \cdots, F_{m}, W_{r+1}, \cdots, W_{n}\right\},
$$

where $\left\{F_{r+1}, \cdots, F_{m}\right\}$ and $\left\{W_{r+1}, \cdots, W_{n}\right\}$ are orthonormal bases of $S(T M)$ and $S\left(T M^{\perp}\right)$ respectively. We use the following range of indices:

$$
\begin{aligned}
& i, j, k, \cdots \in\{1, \cdots, r\}, \quad a, b, c, \cdots \in\{r+1, \cdots, m\}, \\
& \alpha, \beta, \gamma, \cdots \in\{r+1, \cdots, n\}, \quad A, B, C, \cdots \in\{1, \cdots, m\} .
\end{aligned}
$$

Let $P$ be the projection morphism of $T M$ on $S(T M)$ with respect to the decomposition (2.3). Then the local Gauss-Weingartan formulas are given by

$$
\begin{aligned}
& \bar{\nabla}_{X} Y=\nabla_{X} Y+\sum_{i=1}^{r} h_{i}^{\ell}(X, Y) N_{i}+\sum_{\alpha=r+1}^{n} h_{\alpha}^{s}(X, Y) W_{\alpha}, \\
& \bar{\nabla}_{X} N_{i}=-A_{N_{i}} X+\sum_{j=1}^{r} \tau_{i j}(X) N_{j}+\sum_{\alpha=r+1}^{n} \rho_{i \alpha}(X) W_{\alpha},
\end{aligned}
$$




$$
\begin{aligned}
& \bar{\nabla}_{X} W_{\alpha}=-A_{W_{\alpha}} X+\sum_{i=1}^{r} \phi_{\alpha i}(X) N_{i}+\sum_{\beta=r+1}^{n} \theta_{\alpha \beta}(X) W_{\beta}, \\
& \nabla_{X} P Y=\nabla_{X}^{*} P Y+\sum_{i=1}^{r} h_{i}^{*}(X, P Y) \xi_{i}, \\
& \nabla_{X} \xi_{i}=-A_{\xi_{i}}^{*} X-\sum_{j=1}^{r} \sigma_{i j}(X) \xi_{j},
\end{aligned}
$$

for any $X, Y \in \Gamma(T M)$, where $\nabla$ and $\nabla^{*}$ are induced linear connections on $T M$ and $S(T M)$ respectively, the bilinear forms $h_{i}^{\ell}$ and $h_{\alpha}^{s}$ on $M$ are called the local lightlike and local screen second fundamental forms on $T M$ respectively, $h_{i}^{*}$ is called the local second fundamental forms on $S(T M) . A_{N_{i}}, A_{\xi_{i}}^{*}$ and $A_{W_{\alpha}}$ are linear operators on $T M$ and $\tau_{i j}, \rho_{i \alpha}, \phi_{\alpha i}, \theta_{\alpha \beta}$ and $\sigma_{i j}$ are 1-forms on $T M$. We say that

$$
h(X, Y)=\sum_{i=1}^{r} h_{i}^{\ell}(X, Y) N_{i}+\sum_{\alpha=r+1}^{n} h_{\alpha}^{s}(X, Y) W_{\alpha}
$$

is the second fundamental tensor of $M$. Using (2.1), (2.2) and (2.6), we show that

$$
\begin{aligned}
\left(\nabla_{X} g\right)(Y, Z)=- & \pi(Y) g(X, Z)-\pi(Z) g(X, Y) \\
& +\sum_{i=1}^{r}\left\{h_{i}^{\ell}(X, Y) \eta_{i}(Z)+h_{i}^{\ell}(X, Z) \eta_{i}(Y)\right\}, \\
T(X, Y)= & \pi(Y) X-\pi(X) Y, \quad \forall X, Y, Z \in \Gamma(T M),
\end{aligned}
$$

and each $h_{i}^{\ell}$ and $h_{\alpha}^{s}$ are symmetric on $T M$, where $T$ is the torsion tensor with respect to the induced connection $\nabla$ and $\eta_{i}$ is a 1 -form on $T M$ such that

$$
\eta_{i}(X)=\bar{g}\left(X, N_{i}\right), \quad \forall X \in \Gamma(T M), \quad i \in\{1, \cdots, r\} .
$$

From the facts $h_{i}^{\ell}(X, Y)=\bar{g}\left(\bar{\nabla}_{X} Y, \xi_{i}\right)$ and $h_{\alpha}^{s}(X, Y)=\epsilon_{\alpha} \bar{g}\left(\bar{\nabla}_{X} Y, W_{\alpha}\right)$, we know that $h_{i}^{\ell}$ and $h_{\alpha}^{s}$ are independent of the choice of a screen distribution. Note that $\tau_{i j}$ depend on the section $\xi \in \Gamma(\operatorname{Rad}(T M) \mid \mathcal{U})$ and $d\left(\operatorname{tr}\left(\tau_{i j}\right)\right)=d\left(\operatorname{tr}\left(\bar{\tau}_{i j}\right)\right)[4]$.

Taking $Y=\xi_{i}$ to $h_{j}^{\ell}(X, Y)=\bar{g}\left(\bar{\nabla}_{X} Y, \xi_{j}\right)$ and $h_{\alpha}^{s}(X, Y)=\epsilon_{\alpha} \bar{g}\left(\bar{\nabla}_{X} Y, W_{\alpha}\right)$, we get

$$
h_{i}^{\ell}\left(X, \xi_{j}\right)+h_{j}^{\ell}\left(X, \xi_{i}\right)=0, \quad h_{\alpha}^{s}\left(X, \xi_{i}\right)=-\epsilon_{\alpha} \phi_{\alpha i}(X), \quad \forall X \in \Gamma(T M),
$$

due to (2.1) and (2.8). From the first equation of (2.13) [denote $\left.(2.13)_{1}\right]$, we have

$$
h_{i}^{\ell}\left(X, \xi_{i}\right)=0, \quad h_{i}^{\ell}\left(\xi_{j}, \xi_{k}\right)=0, \quad \forall X \in \Gamma(T M) .
$$


The above local second fundamental forms are related to their shape operators by

$$
\begin{gathered}
h_{i}^{\ell}(X, Y)=g\left(A_{\xi_{i}}^{*} X, Y\right)+\lambda_{i} g(X, Y)-\sum_{k=1}^{r} h_{k}^{\ell}\left(X, \xi_{i}\right) \eta_{k}(Y), \\
\bar{g}\left(A_{\xi_{i}}^{*} X, N_{j}\right)=0, \quad \tau_{i j}(X)-\sigma_{j i}(X)=\lambda_{j} \eta_{i}(X), \\
\epsilon_{\alpha} h_{\alpha}^{s}(X, Y)=g\left(A_{W_{\alpha}} X, Y\right)+\epsilon_{\alpha} \nu_{\alpha} g(X, Y)-\sum_{i=1}^{r} \phi_{\alpha i}(X) \eta_{i}(Y), \\
\bar{g}\left(A_{W_{\alpha}} X, N_{i}\right)=\epsilon_{\alpha}\left\{\rho_{i \alpha}(X)-\nu_{\alpha} \eta_{i}(X)\right\}, \\
h_{i}^{*}(X, P Y)=g\left(A_{N_{i}} X, P Y\right)+\mu_{i} g(X, P Y)+\pi(P Y) \eta_{i}(X), \\
\eta_{j}\left(A_{N_{i}} X\right)+\eta_{i}\left(A_{N_{j}} X\right)+\mu_{i} \eta_{j}(X)+\mu_{j} \eta_{i}(X)=0, \\
\epsilon_{\beta} \theta_{\alpha \beta}=-\epsilon_{\alpha} \theta_{\beta \alpha}, \quad \forall X, Y \in \Gamma(T M),
\end{gathered}
$$

where $\epsilon_{\alpha}=\bar{g}\left(W_{\alpha}, W_{\alpha}\right)(= \pm 1)$ denotes the causal character of $W_{\alpha}$, and $\lambda_{i}, \mu_{i}$ and $\nu_{\alpha}$ are smooth functions given by $\lambda_{i}=\pi\left(\xi_{i}\right), \mu_{i}=\pi\left(N_{i}\right)$ and $\nu_{\alpha}=\epsilon_{\alpha} \pi\left(W_{\alpha}\right)$.

Definition 1. A vector field $X$ on a differential manifold $(\mathcal{M}, g)$ with a metric tensor $g$ is said to be conformal Killing on $\mathcal{M}$ if $\mathcal{L}_{X} g=-2 f g$ for any smooth function $f$, where $\mathcal{L}_{X}$ denotes the Lie derivative with respect to $X$, that is,

$$
\left(\mathcal{L}_{X} g\right)(Y, Z)=X(g(Y, Z))-g([X, Y], Z)-g(Y,[X, Z]), \quad \forall Y, Z \in \Gamma(T \mathcal{M}) .
$$

In particular, if $f=0$, then $X$ is called a Killing vector field on $\mathcal{M}$. A distribution $\mathcal{G}$ on $\mathcal{M}$ is called a conformal Killing (or Killing) distribution on $\mathcal{M}$ if each vector field belonging to $\mathcal{G}$ is a conformal Killing (or Killing) vector field on $\mathcal{M}$.

Theorem 2.1. Let $M$ be an r-lightlike submanifold of a semi-Riemannian manifold $\bar{M}$ admitting a semi-symmetric non-metric connection. Then the following assertions are equivalent:

(1) $h_{i}^{\ell}$ vanishes identically on $M$ for all $i$.

(2) $A_{\xi_{i}}^{*}$ satisfies $A_{\xi_{i}}^{*} X=-\lambda_{i} P X$ for all $X \in \Gamma(T M)$ and $i$.

(3) $\operatorname{Rad}(T M)$ is a Killing distribution on $M$.

(4) $\nabla$ is a semi-symmetric non-metric connection on $M$.

Proof. (1) $\Leftrightarrow(2)$ : If $h_{i}^{\ell}=0$ for all $i$, then, using $(2.15)_{1}$ and the fact that $S(T M)$ is non-degenerate, we have $A_{\xi_{i}}^{*} X=-\lambda_{i} P X$ for all $X \in \Gamma(T M)$. Conversely, if $A_{\xi_{i}}^{*} X=-\lambda_{i} P X$ for all $X \in \Gamma(T M)$, then, from $(2.15)_{1}$, we obtain

$$
h_{i}^{\ell}(X, Y)=-\sum_{k=1}^{r} h_{k}^{\ell}\left(X, \xi_{i}\right) \eta_{k}(Y), \quad \forall X, Y \in \Gamma(T M) .
$$


Taking the skew-symmetric part of (2.18) and using $h_{i}^{\ell}$ is symmetric, we get

$$
\sum_{k=1}^{r}\left\{h_{k}^{\ell}\left(X, \xi_{i}\right) \eta_{k}(Y)-h_{k}^{\ell}\left(Y, \xi_{i}\right) \eta_{k}(X)\right\}=0 .
$$

Replacing $Y$ by $\xi_{j}$ to this and using $(2.14)_{2}$, we have $h_{j}^{\ell}\left(X, \xi_{i}\right)=0$ for all $j$ and $i$. From this and (2.18), we have $h_{i}^{\ell}=0$ for all $i$.

$(1) \Leftrightarrow(3)$ : From $(2.11),(2.12)$ and the definition of $\mathcal{L}_{X} g$, we obtain

$$
\begin{aligned}
& \left(\mathcal{L}_{X} g\right)(Y, Z)=g\left(\nabla_{Y} X, Z\right)+g\left(Y, \nabla_{Z} X\right)-2 \pi(X) g(Y, Z) \\
& \quad+\sum_{k=1}^{r}\left\{h_{k}^{\ell}(X, Y) \eta_{k}(Z)+h_{k}^{\ell}(X, Z) \eta_{k}(Y)\right\}, \forall X, Y, Z \in \Gamma(T M) .
\end{aligned}
$$

Taking $X=\xi_{i}$ to this and using (2.10), $(2.15)_{1}$ and $h_{i}^{\ell}$ is symmetric, we have

$$
\left(\mathcal{L}_{\xi_{i}} g\right)(X, Y)=-2 h_{i}^{\ell}(X, Y), \quad \forall X, Y \in \Gamma(T M),
$$

which proves the equivalence of (1) and (3).

(1) $\Leftrightarrow(4)$ : If $h_{i}^{\ell}=0$ for all $i$, then, from (2.11) and (2.12), we have

$$
\left(\nabla_{X} g\right)(Y, Z)=-\pi(Y) g(X, Z)-\pi(Z) g(X, Y), T(X, Y)=\pi(Y) X-\pi(X) Y .
$$

Thus $\nabla$ is a semi-symmetric non-metric connection on $M$. Conversely if $\nabla$ is a semi-symmetric non-metric connection on $M$, from (2.11) we have

$$
\sum_{k=1}^{r}\left\{h_{k}^{\ell}(X, Y) \eta_{k}(Z)+h_{k}^{\ell}(X, Z) \eta_{k}(Y)\right\}=0
$$

for all $X, Y, Z \in \Gamma(T M)$. Replacing $Z$ by $\xi_{i}$ to this, we obtain (2.18). By the method of $(2) \Rightarrow(1)$, we have $h_{i}^{\ell}=0$ for all $i$.

Theorem 2.2. Let $M$ be an r-lightlike submanifold of a semi-Riemannian manifold $\bar{M}$ admitting a semi-symmetric non-metric connection. Then the following assertions are equivalent:

(1) $A_{\xi_{i}}^{*}$ are self-adjoint on $\Gamma(T M)$ with respect to $g$, for all $i$.

(2) $h_{i}^{\ell}$ satisfy $h_{i}^{\ell}\left(X, \xi_{j}\right)=0$ for all $X \in \Gamma(T M)$, $i$ and $j$.

(3) $A_{\xi_{i}}^{*} \xi_{j}=0$ for all $i$ and $j$, i.e., the image of $\operatorname{Rad}(T M)$ with respect to $A_{\xi_{i}}^{*}$ for each $i$ is a trivial vector bundle.

Proof. From $(2.15)_{1}$ and the facts $g$ and $h_{i}^{\ell}$ are symmetric, we have

$$
g\left(A_{\xi_{i}}^{*} X, Y\right)-g\left(X, A_{\xi_{i}}^{*} Y\right)=\sum_{k=1}^{r}\left\{h_{k}^{\ell}\left(X, \xi_{i}\right) \eta_{k}(Y)-h_{k}^{\ell}\left(Y, \xi_{i}\right) \eta_{k}(X)\right\} .
$$


(1) $\Leftrightarrow(2)$. If $h_{i}^{\ell}\left(X, \xi_{j}\right)=0$ for all $X \in \Gamma(T M), i$ and $j$, then we have

$$
g\left(A_{\xi_{i}}^{*} X, Y\right)=g\left(A_{\xi_{i}}^{*} Y, X\right), \quad \forall X, Y \in \Gamma(T M),
$$

for all $X, Y \in \Gamma(T M)$, that is, $A_{\xi_{i}}^{*}$ are self-adjoint with respect to $g$. Conversely, if $A_{\xi_{i}}^{*}$ are self-adjoint with respect to $g$, then we have

$$
\sum_{k=1}^{r}\left\{h_{k}^{\ell}\left(X, \xi_{i}\right) \eta_{k}(Y)-h_{k}^{\ell}\left(Y, \xi_{i}\right) \eta_{k}(X)\right\}=0,
$$

for all $X, Y \in \Gamma(T M)$. Replacing $Y$ by $\xi_{j}$ in this equation and using the second equation of (2.14), we have $h_{j}^{\ell}\left(X, \xi_{i}\right)=0$ for all $X \in \Gamma(T M), i$ and $j$.

$(2) \Leftrightarrow(3)$. Replacing $X$ by $\xi_{j}$ to $(2.15)_{1}$ and using $(2.14)_{2}$, we have

$$
h_{i}^{\ell}\left(X, \xi_{j}\right)=g\left(A_{\xi_{i}}^{*} \xi_{j}, X\right), \quad \forall X \in \Gamma(T M) .
$$

As $S(T M)$ is non-degenerate, we have the equivalence of (2) and (3).

In the sequel, we call self-adjoint $A_{\xi_{i}}^{*}$ the lightlike shape operators of $M$. In this case, we say that $M$ is $\ell$-irrotational. It follows from the equivalence of (1) and (3) in Theorem 2.2 that the radical distribution $\operatorname{Rad}(T M)$ of an $\ell$-irrotational lightlike submanifold $M$ is always integrable and auto-parallel due to (2.10).

Theorem 2.3. Let $M$ be an r-lightlike submanifold of a semi-Riemannian manifold $\bar{M}$ admitting a semi-symmetric non-metric connection. Then the following assertions are equivalent:

(1) $A_{W_{\alpha}}$ are self-adjoint on $\Gamma(T M)$ with respect to $g$ for all $\alpha$.

(2) $h_{\alpha}^{s}$ satisfy $h_{\alpha}^{s}\left(X, \xi_{i}\right)=0$ for all $X \in \Gamma(S(T M))$, $\alpha$ and $i$.

(3) $\phi_{\alpha i}(X)=0$ for all $X \in \Gamma(S(T M))$, $\alpha$ and $i$.

(4) $A_{W_{\alpha}} \xi_{i}=\sum_{j=1}^{r} \epsilon_{\alpha} \rho_{j \alpha}\left(\xi_{i}\right) \xi_{j}$ i.e., the radical distribution $\operatorname{Rad}(T M)$ is invariant vector bundle under the operators $A_{W_{\alpha}}$.

Proof. From $(2.16)_{1}$ and the facts $g$ and $h_{\alpha}^{s}$ are symmetric, we have

$$
g\left(A_{W_{\alpha}} X, Y\right)-g\left(X, A_{W_{\alpha}} Y\right)=\sum_{j=1}^{r}\left\{\phi_{\alpha j}(X) \eta_{j}(Y)-\phi_{\alpha j}(Y) \eta_{j}(X)\right\} .
$$

Replace $Y$ by $\xi_{i}$ in this and using the fact $\phi_{\alpha j}\left(\xi_{i}\right)=\phi_{\alpha i}\left(\xi_{j}\right)$ due to $(2.13)_{2}$, we have $g\left(A_{W_{\alpha}} \xi_{i}, X\right)=-\phi_{\alpha i}(P X)$ for all $X \in \Gamma(T M)$. Also, from (2.19), we have

$$
g\left(A_{W_{\alpha}} P X, P Y\right)-g\left(P X, A_{W_{\alpha}} P Y\right)=g\left(A_{W_{\alpha}} \xi_{j}, \xi_{k}\right)-g\left(\xi_{j}, A_{W_{\alpha}} \xi_{k}\right)=0 .
$$

$(1) \Leftrightarrow(3)$. If $\phi_{\alpha i}(P X)=0$ for all $X \in \Gamma(T M), i$ and $\alpha$, then $g\left(\xi_{i}, A_{W_{\alpha}} X\right)-$ $g\left(A_{W_{\alpha}} \xi_{i}, X\right)=\phi_{\alpha i}(P X)=0$. Thus $g\left(A_{W_{\alpha}} X, Y\right)=g\left(X, A_{W_{\alpha}} Y\right)$ for all $X, Y \in$ 
$\Gamma(T M)$, i.e., $A_{W_{\alpha}}$ are self-adjoint with respect to $g$. Conversely, if $A_{W_{\alpha}}$ are selfadjoint with respect to $g$, then we have $\sum_{k=1}^{r}\left\{\phi_{\alpha k}(X) \eta_{k}(Y)-\phi_{\alpha k}(Y) \eta_{k}(X)\right\}=0$ for all $X, Y \in \Gamma(T M)$. Replace $X$ by $P X$ and $Y$ by $\xi_{i}$ in this equation, we have $\phi_{\alpha i}(P X)=0$ for all $X \in \Gamma(T M), i$ and $\alpha$.

$(2) \Leftrightarrow(3)$. By $(2.13)_{2}$, we have the equivalence of (2) and (3).

$(3) \Leftrightarrow(4)$. If $\phi_{\alpha i}(P X)=0$ for all $X \in \Gamma(T M), i$ and $\alpha$, then $g\left(A_{W_{\alpha}} \xi_{i}, X\right)=0$. From this, we have $P\left(A_{W_{\alpha}} \xi_{i}\right)=0$. In general, since

$$
A_{W_{\alpha}} X=\sum_{i=1}^{r} \epsilon_{\alpha} \rho_{i \alpha}(X) \xi_{i}+P\left(A_{W_{\alpha}} X\right),
$$

we get $A_{W_{\alpha}} \xi_{i}=\sum_{j=1}^{r} \epsilon_{\alpha} \rho_{j \alpha}\left(\xi_{i}\right) \xi_{j}$. Conversely if $A_{W_{\alpha}} \xi_{i}=\sum_{j=1}^{r} \epsilon_{\alpha} \rho_{j \alpha}\left(\xi_{i}\right) \xi_{j}$, from the second equation in this proof, we have $\phi_{\alpha i}(P X)=0$ for all $i$ and $\alpha$.

In the sequel, we call self-adjoint $A_{W_{\alpha}}$ the screen shape operators of $M$. In this case, we say that $M$ is s-irrotational.

Definition 2. An $r$-lightlike submanifold $M$ is called irrotational [8] if $\bar{\nabla}_{X} \xi_{i} \in$ $\Gamma(T M)$ for any $X \in \Gamma(T M)$ and $\xi_{i} \in \Gamma(\operatorname{Rad}(T M))$.

Note that $M$ is irrotational if and only if

$$
h_{j}^{\ell}\left(X, \xi_{i}\right)=0, \quad h_{\alpha}^{s}\left(X, \xi_{i}\right)=\phi_{\alpha i}=0, \quad \forall X \in \Gamma(T M) .
$$

If $M$ is irrotational, then $A_{\xi_{i}}^{*}$ and $A_{W_{\alpha}}$ are the lightlike and screen shape operators of $M$ respectively and $M$ is both $\ell$-irrotational and $s$-irrotational.

Theorem 2.4. Let $M$ be an $r$-lightlike submanifold of a semi-Riemannian manifold $\bar{M}$ admitting a semi-symmetric non-metric connection. If $S\left(T M^{\perp}\right)$ is a conformal Killing distribution on $\bar{M}$, then there exist smooth functions $B_{\alpha} s$ such that

$$
h_{\alpha}^{s}(X, Y)=\epsilon_{\alpha} B_{\alpha} g(X, Y), \quad \forall X, Y \in \Gamma(T M) .
$$

In particular, if $S\left(T M^{\perp}\right)$ is a Killing distribution on $\bar{M}$, then $h_{\alpha}^{s}=0$ for all $\alpha$.

Proof. Using (2.1), (2.2) and the definition of $\overline{\mathcal{L}}_{X} \bar{g}$, we have

$$
\left(\overline{\mathcal{L}}_{X} \bar{g}\right)(Y, Z)=\bar{g}\left(\bar{\nabla}_{Y} X, Z\right)+\bar{g}\left(Y, \bar{\nabla}_{Z} X\right)-2 \pi(X) \bar{g}(Y, Z) .
$$

Using (2.8) and (2.16), we have $\bar{g}\left(\bar{\nabla}_{X} W_{\alpha}, Y\right)=\epsilon_{\alpha}\left\{\nu_{\alpha} g(X, Y)-h_{\alpha}^{s}(X, Y)\right\}$. Thus

$$
\left(\overline{\mathcal{L}}_{W_{\alpha}} \bar{g}\right)(X, Y)=-2 \epsilon_{\alpha} h_{\alpha}^{s}(X, Y), \quad \forall X, Y \in \Gamma(T M) .
$$

From this equation we deduce our assertions. 


\section{Induced Ricci Curvature Tensors}

Denote by $\bar{R}, R$ and $R^{*}$ the curvature tensors of the semi-symmetric non-metric connection $\bar{\nabla}$ on $\bar{M}$, the induced connection $\nabla$ on $M$ and the induced connection $\nabla^{*}$ on $S(T M)$ respectively. Using the Gauss-Weingarten equations for $M$ and $S(T M)$, we obtain the Gauss-Codazzi equations for $M$ and $S(T M)$ :

$$
\begin{aligned}
& \bar{g}(\bar{R}(X, Y) Z, P U)=g(R(X, Y) Z, P U) \\
& +\sum_{i=1}^{r}\left\{h_{i}^{\ell}(X, Z) g\left(A_{N_{i}} Y, P U\right)-h_{i}^{\ell}(Y, Z) g\left(A_{N_{i}} X, P U\right)\right\} \\
& +\sum_{\alpha=r+1}^{n}\left\{h_{\alpha}^{s}(X, Z) g\left(A_{W_{\alpha}} Y, P U\right)-h_{\alpha}^{s}(Y, Z) g\left(A_{W_{\alpha}} X, P U\right)\right\}, \\
& \bar{g}\left(\bar{R}(X, Y) Z, \xi_{i}\right)=\left(\nabla_{X} h_{i}^{\ell}\right)(Y, Z)-\left(\nabla_{Y} h_{i}^{\ell}\right)(X, Z) \\
& -\pi(X) h_{i}^{\ell}(Y, Z)+\pi(Y) h_{i}^{\ell}(X, Z) \\
& +\sum_{k=1}^{r}\left\{\tau_{k i}(X) h_{k}^{\ell}(Y, Z)-\tau_{k i}(Y) h_{k}^{\ell}(X, Z)\right\} \\
& +\sum_{\alpha=r+1}^{n}\left\{\phi_{\alpha i}(X) h_{\alpha}^{s}(Y, Z)-\phi_{\alpha i}(Y) h_{\alpha}^{s}(X, Z)\right\}, \\
& \text { (3.3) } \quad \bar{g}\left(\bar{R}(X, Y) Z, N_{i}\right)=\bar{g}\left(R(X, Y) Z, N_{i}\right) \\
& +\sum_{j=1}^{r}\left\{h_{j}^{\ell}(X, Z) \eta_{i}\left(A_{N_{j}} Y\right)-h_{j}^{\ell}(Y, Z) \eta_{i}\left(A_{N_{j}} X\right)\right\} \\
& +\sum_{\alpha=r+1}^{n}\left\{h_{\alpha}^{s}(X, Z) \eta_{i}\left(A_{W_{\alpha}} Y\right)-h_{\alpha}^{s}(Y, Z) \eta_{i}\left(A_{W_{\alpha}} X\right)\right\}, \\
& \epsilon_{\alpha} \bar{g}\left(\bar{R}(X, Y) Z, W_{\alpha}\right)=\left(\nabla_{X} h_{\alpha}^{s}\right)(Y, Z)-\left(\nabla_{Y} h_{\alpha}^{s}\right)(X, Z) \\
& -\pi(X) h_{\alpha}^{s}(Y, Z)+\pi(Y) h_{\alpha}^{s}(X, Z) \\
& +\sum_{i=1}^{r}\left\{\rho_{i \alpha}(X) h_{i}^{\ell}(Y, Z)-\rho_{i \alpha}(Y) h_{i}^{\ell}(X, Z)\right\} \\
& +\sum_{\beta=r+1}^{n}\left\{\theta_{\beta \alpha}(X) h_{\beta}^{s}(Y, Z)-\theta_{\beta \alpha}(Y) h_{\beta}^{s}(X, Z)\right\}, \\
& \bar{g}\left(\bar{R}(X, Y) N_{i}, \xi_{j}\right)=h_{j}^{\ell}\left(Y, A_{N_{i}} X\right)-h_{j}^{\ell}\left(X, A_{N_{i}} Y\right)+2 d \tau_{i j}(X, Y) \\
& +\sum_{k=1}^{r}\left\{\tau_{i k}(Y) \tau_{k j}(X)-\tau_{i k}(X) \tau_{k j}(Y)\right\}
\end{aligned}
$$




$$
\begin{aligned}
& \quad+\sum_{\alpha=r+1}^{n}\left\{\rho_{i \alpha}(Y) \phi_{\alpha j}(X)-\rho_{i \alpha}(X) \phi_{\alpha j}(Y)\right\} \\
& =h_{i}^{*}\left(X, A_{\xi_{j}}^{*} Y\right)-h_{i}^{*}\left(Y, A_{\xi_{j}}^{*} X\right)+2 d \sigma_{j i}(X, Y) \\
& +\sum_{k=1}^{r}\left\{\sigma_{j k}(X) \sigma_{k i}(Y)-\sigma_{j k}(Y) \sigma_{k i}(X)\right\} \\
& +\sum_{\alpha=r+1}^{n}\left\{\rho_{i \alpha}(Y) \phi_{\alpha j}(X)-\rho_{i \alpha}(X) \phi_{\alpha j}(Y)\right\} \\
& +\sum_{\alpha=r+1}^{n} \nu_{\alpha}\left\{\phi_{\alpha j}(Y) \eta_{i}(X)-\phi_{\alpha j}(X) \eta_{i}(Y)\right\} \\
& +\sum_{k=1}^{r}\left\{h_{k}^{\ell}\left(Y, \xi_{j}\right) \eta_{i}\left(A_{N_{k}} X\right)-h_{k}^{\ell}\left(X, \xi_{j}\right) \eta_{i}\left(A_{N_{k}} Y\right)\right\},
\end{aligned}
$$

(3.6) $\quad \bar{g}(R(X, Y) P Z, P U)=g\left(R^{*}(X, Y) P Z, P U\right)$

$$
+\sum_{i=1}^{r}\left\{h_{i}^{*}(X, P Z) g\left(A_{\xi_{i}}^{*} Y, P U\right)-h_{i}^{*}(Y, P Z) g\left(A_{\xi_{i}}^{*} X, P U\right),\right.
$$

$$
\begin{aligned}
\bar{g}(R(X, Y) P Z, & \left.N_{i}\right)=\left(\nabla_{X} h_{i}^{*}\right)(Y, P Z)-\left(\nabla_{Y} h_{i}^{*}\right)(X, P Z) \\
& -\pi(X) h_{i}^{*}(Y, P Z)+\pi(Y) h_{i}^{*}(X, P Z) \\
& +\sum_{j=1}^{r}\left\{\sigma_{j i}(Y) h_{j}^{*}(X, P Z)-\sigma_{j i}(X) h_{j}^{*}(Y, P Z)\right\} .
\end{aligned}
$$

Let $\overline{R i c}$ be the Ricci tensor of $\bar{M}$ and let $R^{(0,2)}$ denote the induced Ricci type tensor on $M$ given respectively by

$$
\begin{aligned}
\overline{\operatorname{Ric}}(X, Y) & =\operatorname{trace}\{Z \rightarrow \bar{R}(Z, X) Y\}, \quad \forall X, Y \in \Gamma(T \bar{M}), \\
R^{(0,2)}(X, Y) & =\operatorname{trace}\{Z \rightarrow R(Z, X) Y\}, \quad \forall X, Y \in \Gamma(T M) .
\end{aligned}
$$

Using the quasi-orthonormal frame field (2.5), the above equations reduce to

$$
\begin{aligned}
\text { (3.8) } \overline{\operatorname{Ric}}(X, Y)= & \sum_{i=1}^{r} \bar{g}\left(\bar{R}\left(\xi_{i}, X\right) Y, N_{i}\right)+\sum_{a=r+1}^{m} \epsilon_{a} \bar{g}\left(\bar{R}\left(F_{a}, X\right) Y, F_{a}\right) \\
& +\sum_{i=1}^{r} \bar{g}\left(\bar{R}\left(N_{i}, X\right) Y, \xi_{i}\right)+\sum_{\alpha=r+1}^{n} \epsilon_{\alpha} \bar{g}\left(\bar{R}\left(W_{\alpha}, X\right) Y, W_{\alpha}\right), \\
\text { (3.9) } \quad R^{(0,2)}(X, Y)= & \sum_{i=1}^{r} \bar{g}\left(R\left(\xi_{i}, X\right) Y, N_{i}\right)+\sum_{a=r+1}^{m} \epsilon_{a} g\left(R\left(F_{a}, X\right) Y, F_{a}\right),
\end{aligned}
$$


respectively, where $\epsilon_{a}$ denotes the causal character of $F_{a}$. Substituting (3.1) and (3.3) into (3.8) and using (2.13), (2.15) and (2.16), we obtain

$$
\begin{aligned}
R^{(0,2)}(X, Y) & =\overline{\operatorname{Ric}}(X, Y)+\sum_{i=1}^{r} h_{i}^{\ell}(X, Y) \operatorname{tr} A_{N_{i}}+\sum_{\alpha=r+1}^{n} h_{\alpha}^{s}(X, Y) \operatorname{tr} A_{W_{\alpha}} \\
& -\sum_{i=1}^{r} h_{i}^{\ell}\left(A_{N_{i}} X, Y\right)-\sum_{\alpha=r+1}^{n} h_{\alpha}^{s}\left(A_{W_{\alpha}} X, Y\right) \\
& -\sum_{i=1}^{r} \bar{g}\left(\bar{R}\left(N_{i}, X\right) Y, \xi_{i}\right)-\sum_{\alpha=r+1}^{n} \epsilon_{\alpha} \bar{g}\left(\bar{R}\left(W_{\alpha}, X\right) Y, W_{\alpha}\right),
\end{aligned}
$$

for all $X, Y \in \Gamma(T M)$. This shows that $R^{(0,2)}$ is not symmetric. The tensor field $R^{(0,2)}$ is called the induced Ricci tensor [3, 4] of $M$, denoted by Ric, if it is symmetric. $M$ is Ricci flat if its induced Ricci tensor vanishes identically on $M$.

Theorem 3.1. Let $M$ be an $r$-lightlike submanifold of a semi-Riemannian manifold $\bar{M}$ admitting a semi-symmetric non-metric connection $\bar{\nabla}$. Then the Ricci type tensor $R^{(0,2)}$ of the induced connection $\nabla$ on $M$ of $\bar{\nabla}$ is symmetric if and only if the 1-form $d\left(\operatorname{tr}\left(\tau_{i j}\right)\right)$ is closed, i.e., $d\left(\operatorname{tr}\left(\tau_{i j}\right)\right)=0$, on any coordinate neighborhood $\mathcal{U} \subset M$.

Proof. Using (2.16), (2.19), (3.5) 1 , (3.10) and the first Bianchi's identity, we get

$$
R^{(0,2)}(X, Y)-R^{(0,2)}(Y, X)=2 d\left(\operatorname{tr}\left(\tau_{i j}\right)\right)(X, Y), \quad \forall X, Y \in \Gamma(T M) .
$$

From which, we show that $R^{(0,2)}$ is symmetric if and only if $d\left(\operatorname{tr}\left(\tau_{i j}\right)\right)=0$.

Note 1. For any $r$-lightlike submanifold $M$ of a semi-Riemannian manifold $\bar{M}$ admits a Levi-Civita connection, the similar result proved by Duggal and Jin [4].

A semi-Riemannian manifold $\bar{M}$ of constant curvature $c$ is called a space form and denote it by $\bar{M}(c)$. In this case, the curvature tensor $\bar{R}$ of $\bar{M}$ is given by

$$
\bar{R}(X, Y) Z=c\{\bar{g}(Y, Z) X-\bar{g}(X, Z) Y\}, \quad \forall X, Y, Z \in \Gamma(T \bar{M}) .
$$

In case the ambient manifold $\bar{M}$ is a semi-Riemannian space form $\bar{M}(c)$, we have

$$
\begin{aligned}
R^{(0,2)}(X, Y) & =(m-1) c g(X, Y) \\
& +\sum_{i=1}^{r} h_{i}^{\ell}(X, Y) \operatorname{tr} A_{N_{i}}+\sum_{\alpha=r+1}^{n} h_{\alpha}^{s}(X, Y) \operatorname{tr} A_{W_{\alpha}} \\
& -\sum_{i=1}^{r} h_{i}^{\ell}\left(A_{N_{i}} X, Y\right)-\sum_{\alpha=r+1}^{n} h_{\alpha}^{s}\left(A_{W_{\alpha}} X, Y\right),
\end{aligned}
$$

for all $X, Y \in \Gamma(T M)$. 


\section{Tangential Characteristic Vector Field}

In this section, we may assume that $\zeta$ is tangent to $M$. Then we show that $\lambda_{i}=\pi\left(\xi_{i}\right)=0, \nu_{\alpha}=\epsilon_{\alpha} \pi\left(W_{\alpha}\right)=0$ and $\tau_{i j}=\sigma_{j i}$ due to $(2.15)_{3}$, for all $i, j$ and $\alpha$.

Proposition 4.1. Let $M$ be an $r$-lightlike submanifold of a semi-Riemannian manifold $\bar{M}$ admitting a semi-symmetric non-metric connection. If $\zeta$ is tangent to $M$, then there exists a screen distribution $S(T M)$ such that it contains $\zeta$.

Proof. Assume that $\zeta$ belongs to $\operatorname{Rad}(T M)$. Then we have

$$
\zeta=\sum_{i=1}^{r} \mu_{i} \xi_{i}, \quad 1=\bar{g}(\zeta, \zeta)=\sum_{i, j=1}^{r} \mu_{i} \mu_{j} g\left(\xi_{i}, \xi_{j}\right)=0 .
$$

It is a contradiction. Thus $\zeta$ does not belong to $\operatorname{Rad}(T M)$. Due to the decomposition $(2.3)_{1}$, this result enables one to choose a screen distribution $S(T M)$ which contains $\zeta$. We call such a $S(T M)$ the natural screen distribution of $M$.

Note 2. Although $S(T M)$ is not unique, it is canonically isomorphic to the factor vector bundle $S(T M)^{\sharp}=T M / \operatorname{Rad}(T M)$ considered by Kupeli [8]. Thus all $S(T M)$ are mutually isomorphic. For this reason, in this section we consider only $r$-lightlike submanifolds $M$ of a semi-Riemannian manifold $\bar{M}$ admitting a semi-symmetric non-metric connection equipped with a natural screen distribution $S(T M)$.

Theorem 4.1. Let $M$ be an $r$-lightlike submanifold of a semi-Riemannian manifold $\bar{M}$ admitting a semi-symmetric non-metric connection. If the characteristic vector field $\zeta$ is tangent to $M$, then we have

$$
h_{i}^{\ell}(X, \zeta)=0, \quad \forall X \in \Gamma(T M) .
$$

Moreover, if $\zeta$ is tangent to $M$ and $M$ is s-irrotational, then we have

$$
h_{\alpha}^{s}(X, \zeta)=0, \quad \forall X \in \Gamma(T M) .
$$

Proof. Using the equations of (3.5) and the facts $\nu_{\alpha}=0$ and $\tau_{i j}=\sigma_{j i}$, we get

$$
\begin{array}{r}
h_{j}^{\ell}\left(Y, A_{N_{i}} X\right)-h_{j}^{\ell}\left(X, A_{N_{i}} Y\right)=h_{i}^{*}\left(X, A_{\xi_{j}}^{*} Y\right)-h_{i}^{*}\left(Y, A_{\xi_{j}}^{*} X\right) \\
+\sum_{k=1}^{r}\left\{h_{k}^{\ell}\left(Y, \xi_{j}\right) \eta_{i}\left(A_{N_{k}} X\right)-h_{k}^{\ell}\left(X, \xi_{j}\right) \eta_{i}\left(A_{N_{k}} Y\right)\right\},
\end{array}
$$

for all $X, Y \in \Gamma(T M)$. Using $(2.15)_{1},(2.17)_{1,2}$ and the fact $\lambda_{i}=\mu_{i}=0$, we have

$$
\pi\left(A_{\xi_{j}}^{*} X\right) \eta_{i}(Y)=\pi\left(A_{\xi_{j}}^{*} Y\right) \eta_{i}(X), \forall X, Y \in \Gamma(T M) .
$$


Taking $X=\xi_{i}$ and $Y=\zeta$ to $(2.15)_{1}$ and using $(2.14)_{1,2}$, we show that $\pi\left(A_{\xi_{i}}^{*} \xi_{i}\right)=0$ for all $i$. Replacing $Y$ by $\xi_{j}$ to the last equation and using $(2.15)_{1}$, we have

$$
h_{i}^{\ell}(X, \zeta)=\pi\left(A_{\xi_{i}}^{*} X\right)=0, \quad \forall X \in \Gamma(T M) .
$$

Taking the scalar product with $W_{\alpha}$ and $N_{i}$ to the Weingarten equations $\bar{R}(X, Y) N_{i}$ and $\bar{R}(X, Y) W_{\alpha}$ for $M$ respectively, and then, comparing this two results, we get

$$
\begin{aligned}
& \epsilon_{\alpha}\left\{h_{\alpha}^{s}\left(Y, A_{N_{i}} X\right)-h_{\alpha}^{s}\left(X, A_{N_{i}} Y\right)+2 d \rho_{i \alpha}(X, Y)\right. \\
& \quad+\sum_{j=1}^{r}\left[\tau_{i j}(Y) \rho_{j \alpha}(X)-\tau_{i j}(X) \rho_{j \alpha}(Y)\right] \\
& \left.\quad+\sum_{\beta=r+1}^{n}\left[\rho_{i \beta}(Y) \theta_{\beta \alpha}(X)-\rho_{i \beta}(X) \theta_{\beta \alpha}(Y)\right]\right\} \\
& =\bar{g}\left(\nabla_{X}\left(A_{W_{\alpha}} Y\right)-\nabla_{Y}\left(A_{W_{\alpha}} X\right)-A_{W_{\alpha}}[X, Y], N_{i}\right) \\
& \quad+\sum_{j=1}^{r}\left\{\phi_{\alpha j}(Y) \eta_{i}\left(A_{N_{j}} X\right)-\phi_{\alpha j}(X) \eta_{i}\left(A_{N_{j}} Y\right)\right\} \\
& \quad+\sum_{\beta=r+1}^{n}\left\{\theta_{\alpha \beta}(Y) \eta_{i}\left(A_{W_{\beta}} X\right)-\theta_{\alpha \beta}(X) \eta_{i}\left(A_{W_{\beta}} X\right)\right\},
\end{aligned}
$$

for all $X, Y \in \Gamma(T M)$. Using this, $(2.16)_{2}$ and $(2.17)_{3}$, we have

$$
\begin{aligned}
\epsilon_{\alpha}\left\{h_{\alpha}^{s}\left(Y, A_{N_{i}} X\right)-h_{\alpha}^{s}\left(X, A_{N_{i}} Y\right)+2 d \rho_{i \alpha}(X, Y)\right. \\
\left.\quad+\sum_{j=1}^{r}\left[\tau_{i j}(Y) \rho_{j \alpha}(X)-\tau_{i j}(X) \rho_{j \alpha}(Y)\right]\right\} \\
=\bar{g}\left(\nabla_{X}\left(A_{W_{\alpha}} Y\right)-\nabla_{Y}\left(A_{W_{\alpha}} X\right)-A_{W_{\alpha}}[X, Y], N_{i}\right) \\
\quad+\sum_{j=1}^{r}\left\{\phi_{\alpha j}(Y) \eta_{i}\left(A_{N_{j}} X\right)-\phi_{\alpha j}(X) \eta_{i}\left(A_{N_{j}} Y\right\} .\right.
\end{aligned}
$$

Applying $\bar{\nabla}_{X}$ to $\bar{g}\left(A_{W_{\alpha}} Y, N_{i}\right)=\epsilon_{\alpha} \rho_{i \alpha}(Y)$ and using (2.1), (2.7) and (2.8), we get

$$
\begin{aligned}
\bar{g}\left(\nabla_{X}\left(A_{W_{\alpha}} Y\right), N_{i}\right) & =\epsilon_{\alpha} X\left(\rho_{i \alpha}(Y)\right)+\pi\left(A_{W_{\alpha}} Y\right) \eta_{i}(X)+g\left(A_{W_{\alpha}} Y, A_{N_{i}} X\right) \\
& -\sum_{j=1}^{r} \epsilon_{\alpha} \tau_{i j}(X) \rho_{j \alpha}(Y), \quad \forall X, Y \in \Gamma(T M) .
\end{aligned}
$$

Substituting this equation into (4.3) and using $(2.16)_{1}$, we have

$$
\pi\left(A_{W_{\alpha}} X\right) \eta_{i}(Y)=\pi\left(A_{W_{\alpha}} Y\right) \eta_{i}(X), \quad \forall X, Y \in \Gamma(T M) .
$$


Replacing $Y$ by $\xi_{i}$ to this equation and using $\eta_{i}\left(\xi_{i}\right)=1$, we have

$$
\pi\left(A_{W_{\alpha}} X\right)=\pi\left(A_{W_{\alpha}} \xi_{i}\right) \eta_{i}(X), \quad \forall X \in \Gamma(T M) .
$$

As $M$ is $s$-irrotational, taking $X=\xi_{i}$ and $Y=\zeta$ to $(2.16)_{1}$, we get $\pi\left(A_{W_{\alpha}} \xi_{i}\right)=$ $-\phi_{\alpha i}(\zeta)=0$ due to (3) in Theorem 2.3. Replacing $Y$ by $\zeta$ to $(2.16)_{1}$ and using the above result, we have $h_{\alpha}^{s}(X, \zeta)=\pi\left(A_{W_{\alpha}} X\right)=0$ for all $X \in \Gamma(T M)$.

Definition 3. An $r$-lightlike submanifold $M$ of $\bar{M}$ is said to be totally umbilical[4] if there is a smooth vector field $\mathcal{H} \in \Gamma(\operatorname{tr}(T M))$ such that

$$
h(X, Y)=\mathcal{H} g(X, Y), \forall X, Y \in \Gamma(T M) .
$$

In case $\mathcal{H}=0$, we say that $M$ is totally geodesic.

It is easy to see [4] that $M$ is totally umbilical if and only if, on each coordinate neighborhood $\mathcal{U}$, there exist smooth functions $A_{i}$ and $B_{\alpha}$ such that

$$
h_{i}^{\ell}(X, Y)=A_{i} g(X, Y), h_{\alpha}^{s}(X, Y)=B_{\alpha} g(X, Y), \forall X, Y \in \Gamma(T M) .
$$

Corollary 1. Let $M$ be an r-lightlike submanifold of a semi-Riemannian manifold $\bar{M}$ admitting a semi-symmetric non-metric connection such that $\zeta$ is tangent to $M$. If $M$ is totally umbilical, then $M$ is totally geodesic.

Proof. As $M$ is totally umbilical, $M$ is $s$-irrotational by Theorem 2.3. Thus, by Theorem 4.1, we get $h_{i}^{\ell}(X, \zeta)=0$ and $h_{\alpha}^{s}(X, \zeta)=0$ for all $X \in \Gamma(T M)$. From this results and (4.4), we have $A_{i} \pi(X)=0$ and $B_{\alpha} \pi(X)=0$. Replacing $X$ by $\zeta$ to this results, we get $A_{i}=B_{\alpha}=0$ for all $i$ and $\alpha$. Thus $M$ is totally geodesic.

Definition 4. We say that $S(T M)$ is totally umbilical [3] in $M$ if, on any coordinate neighborhood $\mathcal{U} \subset M$, there is a smooth function $\gamma_{i}$ such that

$$
h_{i}^{*}(X, P Y)=C_{i} g(X, Y), \quad \forall X, Y \in \Gamma(T M), i .
$$

In case $C_{i}=0$ on $\mathcal{U}$, we say that $S(T M)$ is totally geodesic in $M$.

Theorem 4.2. Let $M$ be an r-lightlike submanifold of a semi-Riemannian manifold $\bar{M}$ admitting a semi-symmetric non-metric connection such that $\zeta$ is tangent to $M$. If $S(T M)$ is totally umbilical in $M$, then $S(T M)$ is totally geodesic in $M$.

Proof. Taking $i=j$ to $(2.17)_{2}$ and using $\mu_{i}=0$, we have $\bar{g}\left(A_{N_{i}} Y, N_{i}\right)=0$. Applying $\bar{\nabla}_{X}$ to this equation and using $(2.1),(2.6),(2.7)$ and $(2.17)_{2}$, we have

$$
\bar{g}\left(\nabla_{X}\left(A_{N_{i}} Y\right), N_{i}\right)=\pi\left(A_{N_{i}} Y\right) \eta_{i}(X)+g\left(A_{N_{i}} X, A_{N_{i}} Y\right)+\sum_{j=1}^{r} \tau_{i j}(X) \eta_{i}\left(A_{N_{j}} Y\right),
$$


for all $X, Y \in \Gamma(T M)$. Substituting this equation into the following equation

$$
\begin{aligned}
& 0= \bar{g}\left(\bar{R}(X, Y) N_{i}, N_{i}\right) \\
&= \bar{g}\left(-\nabla_{X}\left(A_{N_{i}} Y\right)+\nabla_{Y}\left(A_{N_{i}} X\right)+A_{N_{i}}[X, Y], N_{i}\right) \\
& \quad+\sum_{j=1}^{r}\left\{\tau _ { i j } ( X ) \eta _ { i } \left(A_{N_{j}} Y-\tau_{i j}(Y) \eta_{i}\left(A_{N_{j}} X\right\}\right.\right. \\
& \quad+\sum_{\alpha=r+1}^{n}\left\{\rho_{i \alpha}(X) \eta_{i}\left(A_{W_{\alpha}} Y\right)-\rho_{i \alpha}(Y) \eta_{i}\left(A_{W_{\alpha}} X\right)\right\} \\
&=\quad-\bar{g}\left(\nabla_{X}\left(A_{N_{i}} Y\right), N_{i}\right)+\bar{g}\left(\nabla_{Y}\left(A_{N_{i}} X\right), N_{i}\right) \\
& \quad+\sum_{j=1}^{r}\left\{\tau_{i j}(X) \eta_{i}\left(A_{N_{j}} Y\right)-\tau_{i j}(Y) \eta_{i}\left(A_{N_{j}} X\right)\right\}
\end{aligned}
$$

due to $(2.16)_{2}$ and the fact $\nu_{\alpha}=0$, we have

$$
\pi\left(A_{N_{i}} X\right) \eta_{i}(Y)=\pi\left(A_{N_{i}} Y\right) \eta_{i}(X), \quad \forall X, Y \in \Gamma(T M) .
$$

Replacing $Y$ by $\xi_{j}$ to this equation and using $\eta_{i}\left(\xi_{j}\right)=\delta_{i j}$, we have

$$
\pi\left(A_{N_{j}} X\right)=\pi\left(A_{N_{i}} \xi_{j}\right) \eta_{i}(X), \quad \forall X \in \Gamma(T M) .
$$

As $S(T M)$ is totally umbilical in $M$, replacing $P Y$ by $\zeta$ to $(2.17)_{1}$, we have

$$
\pi\left(A_{N_{j}} X\right)=g\left(A_{N_{j}} X, \zeta\right)=C_{j} \pi(X)-\eta_{j}(X), \pi\left(A_{N_{i}} \xi_{j}\right)=g\left(A_{N_{i}} \xi_{j}, \zeta\right)=-\delta_{i j} .
$$

From the last two results, we have $C_{j} \pi(X)=0$ for any $X \in \Gamma(T M)$. Replacing $X$ by $\zeta$ to this and using $\pi(\zeta)=1$, we get $C_{j}=0$ for all $j$. Thus $S(T M)$ is totally geodesic in $M$.

Theorem 4.3. Let $M$ be an $r$-lightlike submanifold of a semi-Riemannian space form $\bar{M}(c)$ admitting a semi-symmetric non-metric connection such that $\zeta$ is tangent to $M$ and $S(T M)$ is totally umbilical in $M$.

(1) If $M$ is s-irrotational, then we have $c=0$.

(2) If $M$ is $\ell$-irrotational, then $M$ is locally a product manifold $M_{r} \times M_{m-r}$ where $M_{r}$ and $M_{m-r}$ are leaves of the integrable distributions $\operatorname{Rad}(T M)$ and $S(T M)$ respectively.

Proof. (1) As $S(T M)$ is totally umbilical in $M$, we have $h_{i}^{*}=0$ by Theorem 4.2. Thus (3.7) reduce to $\bar{g}\left(R(X, Y) P Z, N_{i}\right)=0$ for all $X, Y, Z \in \Gamma(T M)$. Substituting this result and (3.11) into (3.3) and using $(2.16)_{2}$ with $\nu_{\alpha}=0$, we get 


$$
\begin{aligned}
& c\left\{g(Y, P Z) \eta_{i}(X)-g(X, P Z) \eta_{i}(Y)\right\} \\
& =\sum_{j=1}^{r}\left\{h_{j}^{\ell}(X, P Z) \eta_{i}\left(A_{N_{j}} Y\right)-h_{j}^{\ell}(Y, P Z) \eta_{i}\left(A_{N_{j}} X\right)\right\} \\
& \quad+\sum_{\alpha=r+1}^{n} \epsilon_{\alpha}\left\{h_{\alpha}^{s}(X, P Z) \rho_{i \alpha}(Y)-h_{\alpha}^{s}(Y, P Z) \rho_{i \alpha}(X)\right\} .
\end{aligned}
$$

Taking $X=\xi_{i}$ and $Y=P Z=\zeta$ to this and using (4.1) and (4.2), we have $c=0$.

(2) As $A_{\xi_{i}}^{*}$ is self-adjoint, we have $A_{\xi_{i}}^{*} \xi_{j}=0$ due to (3) in Theorem 2.2. From this and (2.10), we show that $\operatorname{Rad}(T M)$ is an auto-parallel distribution on $M$. As $S(T M)$ is totally umbilical in $M$, we have $h_{i}^{*}=0$ and $\nabla_{X} Y=\nabla_{X}^{*} Y$ for all $X, Y \in \Gamma(S(T M))$ due to (2.9). Thus $S(T M)$ is also an auto-parallel distribution on $M$. By the decomposition theorem of de Rham [2], we have $M=M_{r} \times M_{m-r}$ where $M_{r}$ and $M_{m-r}$ are leaves of $\operatorname{Rad}(T M)$ and $S(T M)$ of $M$ respectively.

Theorem 4.4. Let $M$ be an $r$-lightlike submanifold of a semi-Riemannian manifold $\bar{M}$ admitting a semi-symmetric non-metric connection. If $S\left(T M^{\perp}\right)$ is a conformal Killing distribution on $\bar{M}$, then $S\left(T M^{\perp}\right)$ is a Killing distribution on $\bar{M}$.

Proof. As $S\left(T M^{\perp}\right)$ is a conformal Killing distribution on $\bar{M}$, by (2.21) and (2) in Theorem 2.3, we show that $M$ is $s$-irrotational. Thus we have $h_{\alpha}^{s}(X, \zeta)=0$ for all $X \in \Gamma(T M)$ by Theorem 4.1. Taking $X=Y=\zeta$ to (2.21), we have $B_{\alpha}=0$. Thus $h_{\alpha}^{s}=0$ for all $\alpha$. This implies $S\left(T M^{\perp}\right)$ is a Killing distribution on $\bar{M}$.

Theorem 4.5. Let $M$ be an $\ell$-irrotational $r$-lightlike submanifold of $\bar{M}(c)$ admitting a semi-symmetric non-metric connection such that $\zeta$ is tangent to $M$. If $S(T M)$ is totally umbilical in $M$ and $S\left(T M^{\perp}\right)$ is conformal Killing, then $M$ is Ricci flat.

Proof. As $S(T M)$ is totally umbilical, we have $h_{i}^{*}=0$. From $(2.17)_{1}$, we get

$$
\begin{aligned}
& A_{N_{i}} X=-\eta_{i}(X) \zeta+\sum_{j=1}^{r} \eta_{j}\left(A_{N_{i}} X\right) \xi_{j}, \quad \forall X \in \Gamma(T M), \\
& \operatorname{tr} A_{N_{i}}=\sum_{a=r+1}^{m} \epsilon_{a} g\left(A_{N_{i}} F_{a}, F_{a}\right)+\sum_{k=1}^{r} \bar{g}\left(A_{N_{i}} \xi_{k}, N_{k}\right)=0+\sum_{k=1}^{r} \eta_{k}\left(A_{N_{i}} \xi_{k}\right) .
\end{aligned}
$$

As $S\left(T M^{\perp}\right)$ is conformal Killing, we get $c=0$ and $h_{\alpha}^{s}=0$ by Theorem 4.3 and Theorem 4.4. Also as $M$ is $\ell$-irrotational, $h_{i}^{\ell}\left(X, \xi_{j}\right)=0$. Thus (4.6) reduce to

$$
\sum_{j=1}^{r}\left\{h_{j}^{\ell}(X, Z) \eta_{i}\left(A_{N_{j}} Y\right)-h_{j}^{\ell}(Y, Z) \eta_{i}\left(A_{N_{j}} X\right)\right\}=0,
$$


for all $X, Y, Z \in \Gamma(T M)$. Replacing $Y$ by $\xi_{i}$ to this result, we have

$$
\sum_{j=1}^{r}\left\{h_{j}^{\ell}(X, Y) \eta_{i}\left(A_{N_{j}} \xi_{i}\right)-h_{j}^{\ell}\left(\xi_{i}, Y\right) \eta_{i}\left(A_{N_{j}} X\right)\right\}=0, \quad \forall X, Y \in \Gamma(T M) .
$$

Substituting (4.7) and (4.8) into (3.12) and using (4.9), we have

$$
\begin{aligned}
R^{(0,2)}(X, Y) & =\sum_{i=1}^{r}\left\{h_{i}^{\ell}(X, Y) \operatorname{tr} A_{N_{i}}-h_{i}^{\ell}\left(A_{N_{i}} X, Y\right)\right\} \\
& =\sum_{i, k=1}^{r}\left\{h_{i}^{\ell}(X, Y) \eta_{k}\left(A_{N_{i}} \xi_{k}\right)-h_{i}^{\ell}\left(Y, \xi_{k}\right) \eta_{k}\left(A_{N_{i}} X\right)\right\}=0 .
\end{aligned}
$$

Thus $M$ is Ricci flat.

Theorem 4.6. Let $M$ be an r-lightlike submanifold of a semi-Riemannian space form $\bar{M}(c)$ admitting a semi-symmetric non-metric connection such that $\zeta$ is tangent to $M$. If $S(T M)$ is totally umbilical in $M$ and $S\left(T M^{\perp}\right)$ is a conformal Killing distribution on $\bar{M}$, then the following assertions are equivalent:

(1) $M$ is flat, i.e., the curvature tensor $R$ of $M$ satisfies $R=0$.

(2) The local lightlike second fundamental form $h_{i}^{\ell}$ of $M$ satisfies $h_{i}^{\ell}=0$.

(3) The induced connection $\nabla$ of $M$ is a semi-symmetric non-metric connection.

Proof. First, using (3.1) (3.4), (4.7) and the fact $c=h_{\alpha}^{s}=0$, we show that

$$
\begin{aligned}
R(X, Y) Z= & \sum_{i=1}^{r}\left\{h_{i}^{\ell}(Y, Z) A_{N_{i}} X-h_{i}^{\ell}(X, Z) A_{N_{i}} Y\right\} \\
= & \sum_{i=1}^{r}\left\{h_{i}^{\ell}(X, Z) \eta_{i}(Y)-h_{i}^{\ell}(Y, Z) \eta_{i}(X)\right\} \zeta \\
& +\sum_{i=1}^{r}\left\{h_{i}^{\ell}(Y, Z) \eta_{k}\left(A_{N_{i}} X\right)-h_{i}^{\ell}(X, Z) \eta_{k}\left(A_{N_{i}} Y\right)\right\} \xi_{k} .
\end{aligned}
$$

(1) $\Leftrightarrow(2)$. If $h_{i}^{\ell}=0$ for all $i$, then we have $R=0$. Conversely, if $R=0$, then, taking the scalar product with $\zeta$ to the last equation and using $\lambda_{i}=0$, we have

$$
\sum_{i=1}^{r}\left\{h_{i}^{\ell}(X, Z) \eta_{i}(Y)-h_{i}^{\ell}(Y, Z) \eta_{i}(X)\right\}=0 .
$$

Replacing $Y$ by $\xi_{k}$ to this, we obtain

$$
h_{k}^{\ell}(X, Y)=\sum_{i=1}^{r} h_{i}^{\ell}\left(Y, \xi_{k}\right) \eta_{i}(X)
$$


Taking the skew-symmetric part of this equation, we have

$$
\sum_{k=1}^{r}\left\{h_{k}^{\ell}\left(X, \xi_{i}\right) \eta_{k}(Y)-h_{k}^{\ell}\left(Y, \xi_{i}\right) \eta_{k}(X)\right\}=0 .
$$

Replacing $Y$ by $\xi_{j}$ to this and using $(2.14)_{2}$, we have $h_{j}^{\ell}\left(X, \xi_{i}\right)=0$ for all $X \in$ $\Gamma(T M), i$ and $j$. Substituting this into (4.10), we have $h_{i}^{\ell}=0$ for all $i$.

$(2) \Leftrightarrow(3)$. The equivalence of (2) and (3) follows from Theorem 2.1.

\section{REFERENCES}

1. Ageshe, N.S. \& Chafle, M.R.: A semi-symmetric non-metric connection on a Riemannian manifold. Indian J. Pure Appl. Math. 23 (1992), no. 6, 399-409.

2. de Rham, G.: Sur la réductibilité d'un espace de Riemannian. Comm. Math. Helv. 26 (1952), 328-344.

3. Duggal, K.L. \& Bejancu, A.: Lightlike Submanifolds of Semi-Riemannian Manifolds and Applications. Kluwer Acad. Publishers, Dordrecht, 1996.

4. Duggal, K.L. \& Jin, D.H.: Totally umbilical lightlike submanifolds. Kodai Math. J. 26 (2003), 49-68.

5. Hawking, S.W. \& Ellis, G.F.R.: The large scale structure of space-time. Cambridge University Press, Cambridge, 1973.

6. Jin, D.H.: Geometry of lightlike hypersurfaces of a Lorentz manifold with a semisymmetric non-metric connection. J. Geo. Phy., in press.

7. Jin, D.H. \& Lee, J.W.: Geometry of half lightlike submanifolds of a semi-Riemannian manifold with a semi-symmetric non-metric connection. accepted in Bull. Korean Math. Soc. 2012.

8. Kupeli, D.N.: Singular Semi-Riemannian Geometry, Mathematics and Its Applications. vol. 366, Kluwer Acad. Publishers, Dordrecht, 1996.

9. O'Neill, B.: Semi-Riemannian Geometry with Applications to Relativity. Academic Press, 1983.

10. Yaşar, E., Çöken, A. C. \& Yücesan, A.: Lightlike hypersurfaces in semi-Riemannian manifold with semi-symmetric non-metric connection. Math. Scand. 102 (2008), 253264.

Department of Mathematics, Dongguk University, Gyeonguu 780-714, Republic of KoREA

Email address: jindh@dongguk.ac.kr 